\title{
LA IMPORTANCIA DE LA HISTORIA DE LA QUÍMICA EN LA ENSEÑANZA ESCOLAR: ANÁLISIS DEL PENSAMIENTO Y ELABORACIÓN DE MATERIAL DIDÁCTICO DE PROFESORES EN FORMACIÓN
}

\author{
The importance of the History of Chemistry \\ in school education: analysis of teachers in training \\ process' thought and elaboration of didactic material
}

\author{
Luigi Cuellar Fernández ${ }^{1}$ \\ Mario Quintanilla Gatica ${ }^{2}$ \\ Ainoa Marzàbal Blancafort ${ }^{3}$
}

\begin{abstract}
El reconocimiento de la importancia de incorporar disciplinas metacientíficas en la reflexión sobre la práctica profesional docente es uno de los aspectos en los que se trabaja actualmente en la investigación en Didáctica de las Ciencias. En el presente estudio se analizan diversos factores del pensamiento de un grupo de Profesores en Formación en relación a las implicaciones didácticas de incluir la historia de la Química en las secuencias de enseñanza. En un orden progresivo de autonomía, en la primera fase se valora a nivel teórico, en la segunda se analizan materiales con diferentes enfoques didácticos, y en la tercera fase se crean materiales inéditos. En la cuarta fase se analiza el grado de coherencia en las tres fases anteriores, y se elige una muestra representativa entre los participantes, a los que se entrevista en la quinta fase.
\end{abstract}

Palabras clave: Historia de la ciencia. Naturaleza de la ciencia. Modelos científicos. Concepciones docentes. Formación docente.

\begin{abstract}
The recognition of the importance of incorporating metascientific disciplines in the professional educational practice is one of the aspects in which the research in Didactics of the Sciences is focusing nowadays. In this study we analyze several factors found in the thought of a group of teachers in relation to the didactic implications of including the history of the Chemistry in the sequences of education. In a progressive order of autonomy, in the first phase it is valued to theoretical level, in the second one, materials are analyzed by different didactic approaches, and in the third phase they create their own materials. In the fourth phase we analyze the coherence degree in the previous phases, and a representative sample is chosen among the participants, to be interviewed in the fifth phase.
\end{abstract}

Keywords: History of science. Nature of science. Scientific models. Teachers conceptions. Teacher training.

\footnotetext{
${ }^{1}$ Licenciado en Química, doctor en Ciencias de la Educación. Departamento de Didáctica, Universidad Católica de la Santísima Concepción (UCSC). Concepción, Chile. <lcuellar@ucsc.cl>.

${ }^{2}$ Licenciado en Biologia y Química, doctor en Didáctica de las Ciencias Experimentales y las Matemáticas. Facultad de Educación, Pontificia Universidad Católica de Chile. Santiago de Chile. <mquintag@uc.cl> ${ }^{3}$ Licenciada en Química, doctora (c) en Didáctica de las Ciencias Experimentales y las Matemáticas. Departamento de Didáctica, UCSC. Concepción, Chile.<ainoamb@hotmail.com>
}

${ }^{1}$ Alonso de Ribera, 2850

Concepción, Chile 
Fernández, L C.; Gatica, M. Q.; Blancafort, A. M.

\section{Introducción}

Uno de los aspectos fundamentales para mejorar la calidad de la educación científica tiene que ver con la formación de los futuros profesores especialistas en Educación Básica, cuyo déficit actualmente se sitúa en el componente disciplinar específico, en áreas como las matemáticas y las ciencias naturales. Es así como en Chile se han iniciado ya la formulación de varios Proyectos a nivel nacional, de mejoramiento de la calidad de la Educación en Ciencias, entre los cuales se citan aquellos que se orientan en los niveles básicos de formación ${ }^{4}$. De esta forma, aparece como eje fundamental en la formación docente el conocimiento disciplinar, el conocimiento didáctico de la disciplina y la formación en competencias de pensamiento científico (CPC), algo ya reconocido a nivel nacional e internacional a partir de múltiples Proyectos y estudios (PORLÁN y MARTÍN DEL POZO, 2006).

En este marco de renovación, entendemos que en la enseñanza de las ciencias es pertinente el abordaje del conocimiento científico desde una perspectiva naturalizada (GIERE, 1992; QUINTANILLA, 2006) en la que resulta fundamental la adquisición del lenguaje propio de la actividad científica, que promueva actitudes científicas en los estudiantes, lo cual puede lograrse desde la enseñanza de la química a partir de su perspectiva histórica, en la que se aborde la ontogénesis y filogénesis de cada uno de los cuerpos conceptuales de esta ciencia. Por otra parte, está bien fundamentada teóricamente la importancia de esta disciplina metacientífica en la formación de profesores (MATTHEWS, 1994; QUINTANILLA, IZQUIERDO y ADÚRIZ-BRAVO, 2005). No obstante, consideramos necesario el adelantar estudios que conlleven a recabar evidencia empírica acerca de las implicancias que conlleva la incorporación de esta perspectiva histórica de la ciencia, como eje articulador del discurso químico de los profesores en ejercicio y en formación para este caso particular.

De esta forma, se planteó como objetivo central, analizary valorar la importancia concedida a la Historia de la Química en la elaboración de material bibliográfico, por parte de profesores de Enseñanza general Básica (EGB) en formación, para el abordaje teóricamente fundamentado de nociones científicas en el aula. Para ello, inicialmente se tomó registro de las concepciones de los participantes en torno a la naturaleza y la historia de la ciencia. A continuación se presento la temática referida a la teoría atómica por medio de materiales con distintos enfoques, tradicionalteórico e histórico, discutiendo con los participantes su pertinencia. Después se propuso la elaboración de materiales curriculares inéditos, en relación a la ley periódica.

El análisis de los datos recogidos nos permite identificar y caracterizar las concepciones previas de los futuros profesores como punto de partida, así como la coherencia entre su posicionamiento teórico y la generación de sus propias propuestas. Para profundizar en la tipología de casos encontrados se eligió una muestra representativa de participantes, con quienes se adelantó una entrevista.

\footnotetext{
${ }^{4} \mathrm{Al}$ respecto se pueden consultar los proyectos financiados por el Programa de Mejoramiento a la Calidad de la Educación Superior - MECESUP-, en particular al Proyecto "Renovación de las Pedagogías y los Programas de continuidad, en el marco del Mecesup2 Bicentenario (2004-2008).
} 
La importancia de la historia ...

\section{Desarrollo}

Como lo hemos planteado en otros artículos y producciones científicas (QUINTANILLA, 2006; CUELLAR y QUINTANILLA, 2008) es necesario que las prácticas docentes de educación química posibiliten al estudiantado comprender el indudable carácter bistórico de la química, es decir, la idea de que el conocimiento científico 'está vivo' aunque esté escrito en los libros, que la química como ciencia es dinámica y progresivamente mutable, que los conceptos, modelos y teorías científicas que constituyen el entramado de la química terminan siendo sustituidos por otros, y que los marcos ideológicos que fundamentan el conocimiento en cada época sufren igualmente un proceso de cambio conceptual o paradigmático natural, sistemático, continuo e irreversible, que puede ser comprendido a la luz de ciertos principios teóricos y caracterizado con criterios metodológicos específicos (TOULMIN, 1977; CAMACHO, 2005).

Sin embargo, en múltiples ocasiones se soslaya que para lo planteado anteriormente es necesaria una profunda reflexión teórica y metodológica del discurso químico, ya que el modelo de ciencia define qué contenidos se enseñan y bajo qué enfoque didáctico (IZQUIERDO y ALIBERAS, 2004). Es por esto que cuando nos referimos a una mayor formación en el componente disciplinar específico, resaltamos los aspectos epistemológicos y didácticos que hacen parte de los diversos modelos de formación de los profesores que divulgan el conocimiento químico en la sala de clase.

Para promover una reflexión en este sentido consideramos que el componente histórico en la formación de los profesores de ciencias, posibilita algunos objetivos relacionados con una mayor comprensión de la actividad cientifica, de comprensión profunda de los significados de los modelos teóricos y sus conceptos asociados; posibilidad de establecer relaciones metateóricas con la filosofía y la sociología de la ciencia; servir de puente entre las disciplinas científicas y las humanidades, valoración de los modelos cientificos vigentes en tanto construcciones teóricas sistemáticamente progresivas, desde una perspectiva naturalizada (KRAGH, 1989). De esta forma, no cabe duda del papel fundamental de la Historia de la ciencia en su enseñanza, en tanto que contribuye al cuestionamiento de la habitual visión dogmática de la ciencia y sus métodos.

\section{Una breve reflexión entorno a la Historia de la Ciencia}

No obstante lo anterior no es posible pensar en una simplicidad ingenua al hablar de la Historia de la Ciencia, pues como comentaremos brevemente a continuación existen múltiples formas de interpretarla.

A este respecto Kragh (1989) presenta un análisis interesante que permite identificar las diferentes valoraciones e interpretaciones que pueden atribuirse a la Historia de la Ciencia según la época y el "lente" desde la cual esta sea concebida.

En principio, se resalta que en el desarrollo de la ciencia, las descripciones y los análisis históricos han ido siempre a la saga del desarrollo de la ciencia y no obstante esto, su reconocimiento como disciplina de estudio no emerge hasta el siglo XX. Así, hasta la Edad Media, la forma habitual del desarrollo científico implicaba la referencia de la antigüedad clásica, lo cual significaba cierto status. Durante los siglos XVI y principios del siglo XVII, la Historia, y 
sobre todo la Historia de la antigüedad, eran consideradas por los pioneros, desde Copérnico a Harvey, como algo definitivamente presente en el progreso de la ciencia. Durante la revolución científica la Historia como apoyo en las discusiones ideológicas sería la legitimación de la ciencia. Sin embargo, durante el siglo XVII se presenta un cambio de actitud frente a estas autoridades clásicas, debido a la influencia del Protestantismo y la crítica a los eruditos de la antigua Grecia por ser considerados paganos. De esta forma, emerge una relación de la ciencia con los conocimientos bíblicos, que databan de una época anterior a los griegos. Durante esta época cada ciencia fue adquiriendo valor y autoridad por sí misma, se hizo menos necesario recurrir a la antigüedad como medio de validación.

En el siglo XVIII, para Priestley y sus contemporáneos la Historia de la Ciencia era primordialmente un instrumento cuyo valor se hallaba ligado al progreso de las investigaciones que se llevan a cabo en la época. Se señala que a partir de la actitud de conocer lo relativo a los descubrimientos y avances científicos, la Historia de la Ciencia tomó los rasgos de la Historia del progreso, de lo cual puede derivar una perspectiva ligada a la Historia triunfalista, que no tiene en cuenta las aportaciones que han sido superadas.

Durante los siglos XVII y XVIII la palabra "histórico" no significaba lo que hoy día. Un fenómeno "histórico" significaba frecuentemente un fenómeno concreto, objetivo, e "Historia" significaba simplemente una relación de las condiciones objetivas, sin que fuera necesario que pertenecieran al pasado. El siglo XVIII, por su parte, se caracterizó por una tendencia "antihistórica". La Ilustración veía la Historia como un instrumento del progreso en su lucha contra el orden feudal; desde una perspectiva presentista, sólo los desarrollos recientes merecían interés, mientras que el pasado se consideraba, por lo general, irracional e inferior. Este periodo se caracterizó por una falta de conciencia histórica, consecuencia de las ideas dominantes en torno al conocimiento, en particular a las ideas racionalistas de Descartes, para quien el conocimiento era puramente reflexivo y racional, una abstracción universal y ahistórica.

A finales del siglo XVIII, la corriente Romántica otorgaba un sentido más profundo a la Historia basada en la idea de que al pasado se le debía juzgar según sus propias premisas y no bajo el pensamiento contemporáneo como habían hecho los ilustrados. Así se da un reconocimiento a lo que actualmente podemos considerar como historiografía diacrónica (KRAGH, 1989).

No obstante, durante el siglo XIX, en periodos de progreso, consolidación y organización de la vida científica, se presentó un distanciamiento entre las ciencias naturales y las humanidades. El auge positivista de la ciencia del siglo XIX, sus seguidores, los métodos y las posibilidades hicieron ahistórica a la Historia de la Ciencia, considerando inequívocos y universales sus métodos. La perspectiva histórica se rechazó y el interés se centró entonces en la ciencia contemporánea y sus inmediatos predecesores, resaltando que la Historia era una disciplina humanística, cuyos métodos y objetivos eran incompatibles con los de las ciencias naturales. Esta distinción hizo que se ignorara la Historia de la Ciencia relegándola a los científicos y a los historiadores aficionados.

Para el análisis que nos interesa cabe resaltar que en esta época se escribió la Historia de la Ciencia bajo intereses patrióticos, para resaltar la ciencia de las naciones. Estas obras, en principio diseminadas, comenzaron a organizarse a principios del nuevo siglo $(\mathrm{XX})$ y fue así como la Historia de la Ciencia empezó a asentarse como una disciplina independiente, caracterizada por congresos y conferencias internacionales y por el establecimiento de sociedades nacionales para su estudio. 
La importancia de la historia ...

Finalmente a lo largo del siglo XX la Historia de la Ciencia se ha convertido en objeto de creciente interés debido a su contribución a la Historia y a su valor educativo, y en las últimas décadas ha despertado interés creciente en la comunidad científica de historiadores, sociólogos y científicos, particularmente en los didactas de la ciencia.

\section{¿Qué Historia de la Química han de conocer y enseñar los docentes?}

Algunos autores (FURIÓ, 1996; GIL, CARRASCOSA y MARTÍNEZ-TERRADES, 1999; IZQUIERDO, 2000; SANMARTÍ, 2002. GARCÍA, 2003) consideran que una de las principales dificultades que se presentan en la enseñanza de la química, y que pueden llegar a tener directa relación con los bajos resultados académicos de los estudiantes en esta área, es el carácter tradicionalmente transmisionista, algorítmico y absolutista, con el cual el profesor hace de esta disciplina el objeto de estudio en el aula. Por esta razón, se considera que la enseñanza de la química basada en una perspectiva histórica, en la que adquiere importancia el diseño de unidades didácticas que incorporen los aspectos involucrados en la génesis y evolución de los conceptos científicos, permitirá dimensionar el carácter problematizador de la enseñanza y el aprendizaje de las ciencias en busca del cambio en la imagen de ciencia que generalmente se socializa en el aula y no contempla el propio proceso histórico del sujeto que aprende ciencias (LABARRERE y QUINTANILLA, 2002).

Si en algo estamos de acuerdo quienes nos dedicamos a la investigación en didáctica de las ciencias experimentales, es que la química es un proceso de constitución del saber erudito con dimensiones sociales, valóricas, políticas y culturales. Esta idea de formación y enseñanza de las ciencias naturales desde una orientación de ciudadanía y valores, permite releer y comprender permanentemente marcos teóricos diversos para interpretar fenómenos científicos que boy comprendemos bien y que se explican mediante teorías vigentes, las cuales continúan evolucionando vertiginosamente. Esta perspectiva nos permite además conocer la relación entre la ciencia y la cultura de una época específica, analizando de esta forma la influencia de éstas en el desarrollo y consolidación de una sociedad determinada que comparte unos valores que se resignifican sistemáticamente (BARONA, 1994; SOLSONA, 1997; SOLSONA y QUINTANILLA, 2005; QUINTANILLA et al., 2007). Para dar una respuesta racional, razonable y coherente en este sentido, la hipótesis que sustentamos, es que se plantee el origen histórico, controversial y polémico de las principales teorías de la química, se muestre el proceso de creación y desarrollo de los principales conceptos y metodologías científicas como fruto de un trabajo colectivo y de una construcción humana, en la que hay intrigas, tensiones y distensiones, y se analice así la complejidad de las relaciones ciencia - tecnología - sociedad - comunicación (CTSC) a lo largo de la Historia, con las implicaciones de transformación de los procesos sociales y de convivencia que ello ha generado para la comunidad científica en general y para la comunidad de los químicos en particular (IZQUIERDO et al., 2006).

Así pues es fundamental reconocer el papel del profesor como mediador entre la evolución histórica del conocimiento científico y el conocimiento científico escolar, razón por la cual, se reconoce que la enseñanza de las ciencias y la formación docente han de considerar una resignificación de las bases que los sustentan, para así tratar de formular nuevas propuestas, como la inclusión de la Historia de la Ciencia en la formación del profesorado, tendientes 
Fernández, L C.; Gatica, M. Q.; Blancafort, A. M.

a posibilitar una mayor participación del docente de química en la construcción de su propio conocimiento profesional y científico.

Como se ha podido establecer de forma introductoria, existen múltiples formas de entender la Historia de las Ciencias (HC), motivo por el cual se hace también necesario que el docente identifique las posibles formas de comprenderla, no de manera "genérica" sino que por el contrario, identifique que existe una gran variedad de formas de entenderla, lo cual implica que cada una de estas formas de Historia de las Ciencias, le hace situarse de forma específica y plantear actividades intencionadas, que incluso pueden perseguir objetivos diferentes en el interior del aula.

Como plantean Izquierdo et al (2006) es fundamental considerar el concepto Historia para comprender la imposibilidad de presentar una Historia "totalmente objetiva", ante lo cual se hace necesario que, a partir de las diversas fuentes existentes se elaboren posibles combinaciones y sus respectivas interpretaciones. De lo anterior se pueden originar diversas formas de abordar la Historia de la Ciencia, como pueden ser las perspectivas vertical, horizontal, interna, externa, diacrónica, sincrónica, recurrente, biográfica, entre otras, de manera intencionada según la finalidad de la secuencia didáctica. Así, el profesor de ciencias puede orientar su enseñanza y abordar con sus estudiantes cada una de las teorías o modelos científicos.

\section{Metodología}

En el estudio adelantado se trabajó en cinco fases (Figura 1), con la participación de 74 estudiantes, divididos en dos grupos, de IV año de Formación como Profesores de Enseñanza General Básica.

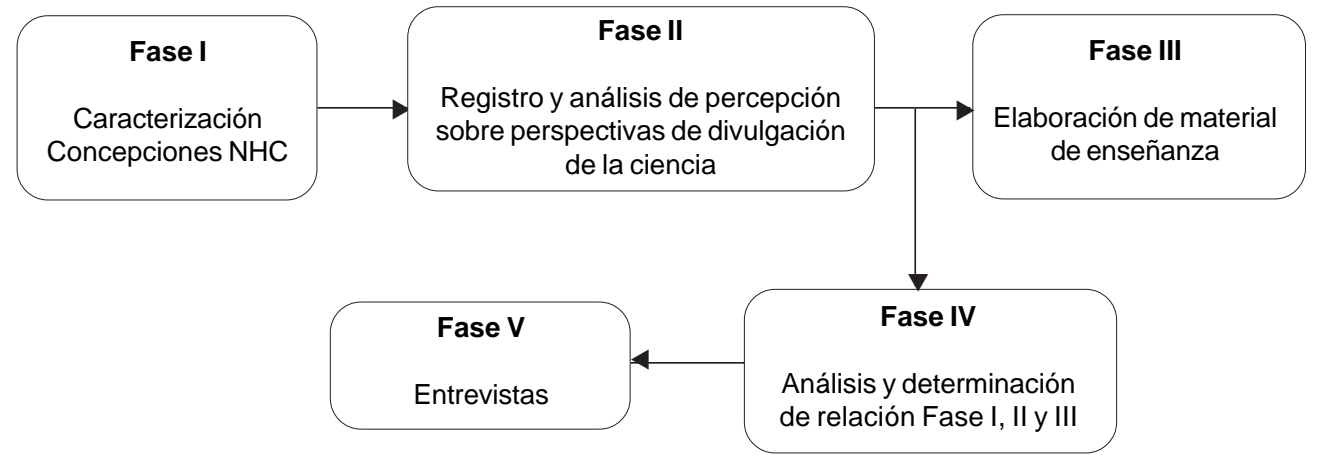

Figura 1. Las diferentes fases en las que se basó el estudio en torno al análisis del pensamiento y la elaboración de material de enseñanza de la Química. 
La importancia de la historia ...

En la Fase I, se ha tomado registro de las concepciones que los profesores en formación tienen acerca de la Naturaleza de la Ciencia (NC) y de la Historia de la Ciencia (HC), para lo cual se ha utilizado un instrumento tipo Likert (cuestionario que hace parte del Proyecto Fondecyt 1095149), a partir del cual se tomó especial énfasis en estas dos dimensiones, $\mathrm{HC}$ y NC.

En la Fase II, se trabajaron los contenidos científicos relacionados con la teoría atómica, la cual fue abordada desde referentes teóricos correspondientes a dos perspectivas diferentes (perspectiva tradicional, PT, ligada a lo conceptual y una perspectiva histórica de la ciencia, PH). Para cada uno de estos dos referentes se incluyó la presentación temática del contenido y sus conceptos asociados, diversas actividades de enseñanza y planteamiento de preguntas, de manera diferenciada, correspondientes a la naturaleza de cada perspectiva.

En la Fase III, se elaboró material bibliográfico, de su propia producción, a modo de “unidades didácticas" para el abordaje de los contenidos científicos relacionados con la Ley Periódica, los cuales serían utilizados en sus propias prácticas profesionales.

En este punto, Fase IV, se hizo el análisis correspondiente a la información recabada en las fases anteriores, con el fin de determinar la coherencia o no, y sus causas, con lo que inicialmente se consideró como subsidio de cada una de las perspectivas de abordaje del conocimiento trabajadas y a las cuales hicieron adscripción.

A partir de los resultados obtenidos, se consideró pertinente adelantar una Fase $V$, que consistió en una entrevista semiestructurada, realizada a algunos de los participantes, teniendo en cuenta que estuvieran representados todos los posicionamientos encontrados, es decir, aquellos en los que se mantuvo coherencia en cualquiera de las tendencias, PT o PH, y aquellos en los que hubo cambios en estas perspectivas, con el fin de indagar en profundidad acerca de las razones por las cuales los profesores en formación habían producido su material bibliográfico desde perspectivas específicas.

\section{Resultados y análisis}

Consideramos pertinente plantear los hallazgos encontrados en cada una de las fases, lo cual permitirá establecer un análisis detallado de lo que progresivamente se iba desarrollando en el marco de esta investigación.

Así las cosas, en relación a la Fase I, que hace referencia a las concepciones que tienen los profesores en formación en torno a la Naturaleza de la actividad cientifica se pudo caracterizar que se configuran tres modelos de ciencia: un modelo tradicional, uno de ciencia como construcción social y uno híbrido - de transición - entre los dos (Figura 2)

Lo anterior pudo establecerse a partir de la manifestación explícita que denota una coexistencia de diversas formas de concebir la naturaleza de la actividad científica (Tabla 1) lo cual se establece en relación a que un gran número de profesores en formación siguen teniendo una visión tradicional de la ciencia, aunque también aparece una evolución hacia una visión más social que todavía no está consolidada. 


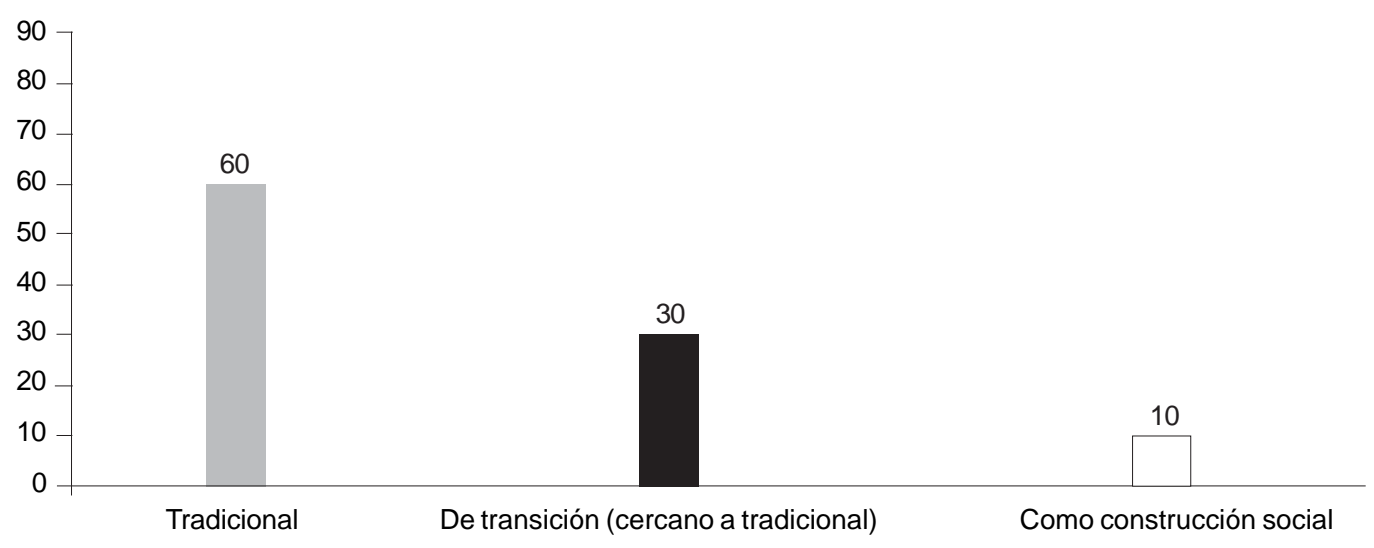

Figura 2. Fase I, Caracterización de los modelos de ciencia, por parte de los participantes.

Tabla 1. categorización de las respuestas de los participantes en torno a la dimensión naturaleza de la ciencia.

\begin{tabular}{|c|c|}
\hline Modelo de ciencia & Caracterización \\
\hline Tradicional & $\begin{array}{l}\text { Se concibe la actividad científica ligada a un método científico, como una estructura } \\
\text { cerrada que no permite usar la creatividad, al servicio de una ciencia objetiva y } \\
\text { estática. }\end{array}$ \\
\hline Construcción social & $\begin{array}{l}\text { Se concibe la ciencia como una actividad que recurre a una metodología de } \\
\text { investigación, que permite usar la creatividad, con alto grado de subjetividad. Se } \\
\text { considera que el profesor debe adoptar un modelo de ciencia y de enseñanza } \\
\text { fundamentados teóricamente. }\end{array}$ \\
\hline Híbrido o de transición & $\begin{array}{l}\text { Parece claro que se alejan de la visión tradicional de la ciencia, pero tienen muchas } \\
\text { incoherencias en sus planteamientos, o bien preguntas sin responder, lo que se } \\
\text { podría interpretar como una falta de reflexión al respecto. }\end{array}$ \\
\hline
\end{tabular}

En lo referido a la dimensión Historia de la Ciencia, se observó una mayor homogeneidad en cuanto a la opinión de los participantes del estudio, ya que para una amplia mayoría la Historia de las Ciencias en la enseñanza puede tener múltiples utilidades y ventajas. Parece que en cierto modo la Historia de la Ciencia puede mejorar la comprensión de los alumnos, hacer que el aprendizaje sea más significativo y que se tenga una visión más humana de la ciencia y de su avance. No obstante lo anterior, hay dos aspectos que llaman la atención:

. Se percibe una gran contradicción con respecto al modelo de ciencia -predominante - que hemos hallado en el apartado anterior, donde la ciencia era considerada mayoritariamente como una disciplina muy estática,

. No se incide en la cuestión fundamental que representa el cómo incorporar la Historia de la Ciencia en la sala de clase.

En relación a la Fase II, en la cual se presentaba el contenido "teoría atómica" desde dos perspectivas teóricas diferentes, una tradicional (PT) basada en lo que se divulga en los libros de química habitualmente utilizados en el curso de ciencias naturales II, y otra con una perspectiva histórica $(\mathbf{P H})$ en el abordaje de los contenidos químicos. Se pudo establecer que 
La importancia de la historia ...

los dos grupos, los cuales presentan una formación teórica antecedente similar (pues pertenecen al mismo Programa de Formación) presentaron resultados diferentes, según se muestra a continuación (Figura 3).

Grupo 01. Análisis perspectivas PT y PH (en \%)
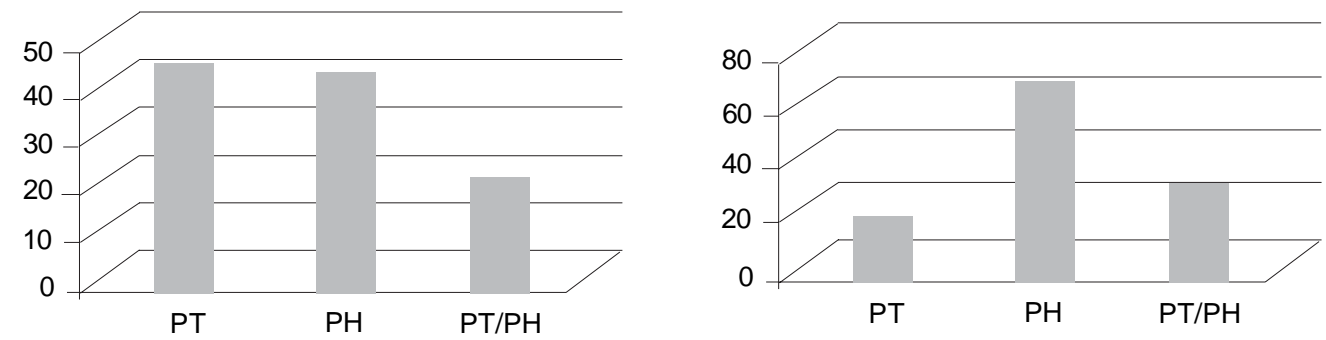

Figura 3. Fase II, adscripción de los profesores en formación a cada una de las perspectivas de abordaje de la química, consideradas en este estudio (PT y PH).

En el grupo 01 es interesante observar cómo al momento de manifestar posicionamiento frente a una de las dos posturas (PT o PH), se encontró que existía cierta equivalencia, siendo un $42 \%$ inclinado a la $\mathbf{P T}$ y un $40 \%$ a la $\mathbf{P H}$. Hubo un importante número de estudiantes, $18 \%$, que manifestaron las ventajas de incorporar ambas perspectivas en el material de enseñanza de la química.

En el grupo 02, se establece que el $12,5 \%$ se inclinaba hacia la PT, un $62.5 \%$ de los estudiantes lo hacía por la $\mathbf{P H}$ y un $25 \%$ consideraba que ambas perspectivas deberían estar presentes en la elaboración del material de enseñanza de la química.

En lo que respecta a la Fase III, y la propia producción de material bibliográfico, en relación al contenido de la Ley Periódica, en la que se propuso diseñar una unidad didáctica para el abordaje de esta temática, cabe resaltar que es el momento en el cual empiezan a develarse las profundas inconsistencias que fueron detectadas en la primera fase de este estudio (Figura 4).

Grupo 01. Produción bibliográfica propia (en \%)

Grupo 02. Produción bibliográfica propia (en \%)
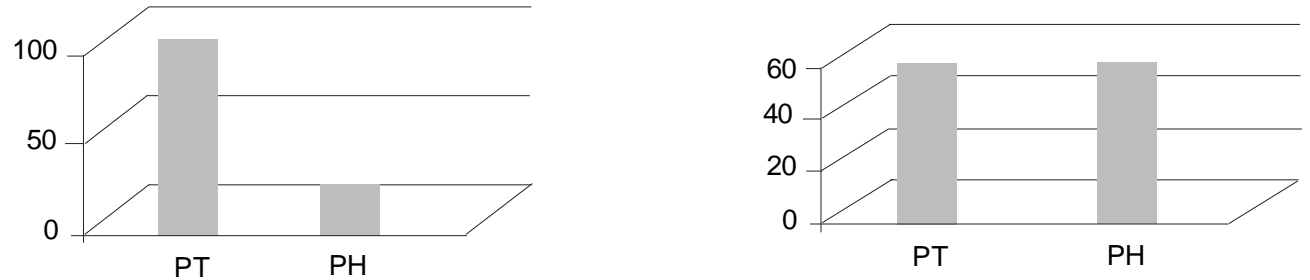

Figura 4. Fase III, elaboración de material bibliográfico inédito, contenido temático Ley Periódica. 
Los estudiantes pertenecientes al Grupo 01, en la elaboración de sus unidades didácticas, relacionadas con la Ley periódica, el $\mathbf{9 1 \%}$ de ellos optó por la perspectiva tradicionalteórica (PT) y solo un 9\% mantuvo intenciones de abordar de forma explícita el componente histórico en el abordaje del tema. Vale decir que de forma intencionada hubo que considerar en este punto la mención a una perspectiva biográfica de la ciencia como la perspectiva histórica, ya que esto fue lo que se encontró en las producciones de los profesores en formación en ambos grupos.

Con respecto al Grupo 02, el 50\% de los participantes se enfocaron por la perspectiva tradicional-teórica (PT) y el restante 50\% lo hizo por la Perspectiva histórica (PH) (la mencionada en el párrafo anterior). Aquellos estudiantes que habían señalado la importancia de presentar ambas perspectivas no fueron consecuentes con su pensamiento en torno a ello.

En este punto correspondiente a la Fase IV, de análisis y determinación de las relaciones encontradas hasta ahora en las fases anteriores, a manera de síntesis de lo comentado en los párrafos precedentes presentamos la Figura 5.

Perspectiva teórica (en \%)

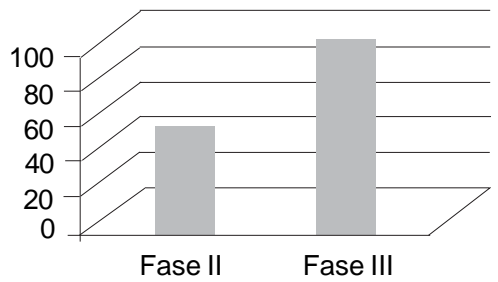

Perspectiva histórica (en \%)

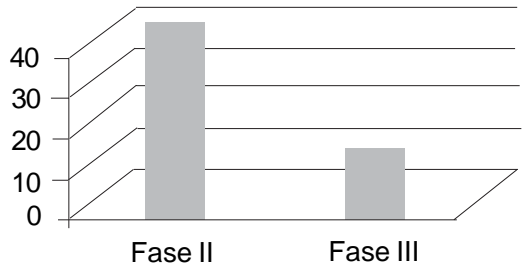

PT/PH en paralelo (en \%)

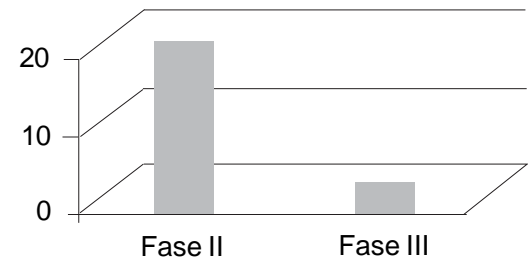

Figura 5. Grupo 01: comparación entre lo declarado en la Fase II y la producción de material en la Fase III:

Se puede observar que para los estudiantes de este Grupo 01 del estudio, hubo un gran aumento hacia la producción de material con base en una PT. De la misma forma, entre aquellos que habían declarado ventajas a la inclusión de la PH en el material de enseñanza, al momento de elaborar su propio material lo hicieron sin tener en cuenta esta PH. Por su parte el grupo que había señalado ventajas de trabajar en forma paralela las dos perspectivas (PT y 
La importancia de la historia ...

$\mathrm{PH}$ ) optaron por hacer su propio material desde la PT, evidenciado esto en el aumento de estas producciones al 91\% del total del Grupo 01 (Figura 6).

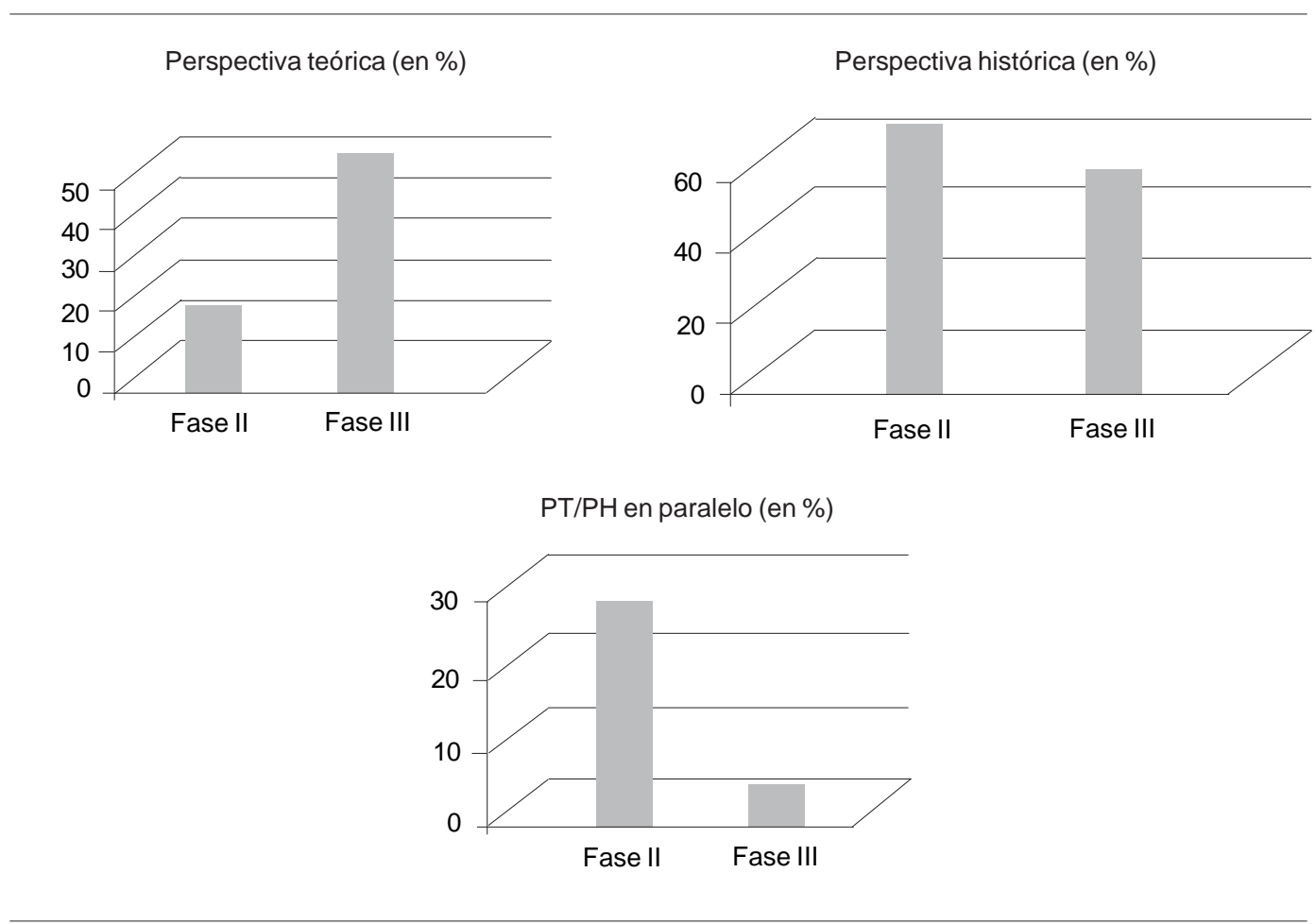

Figura 6. Grupo 02: Comparación entre lo declarado en la Fase Il y la producción de material en la Fase III.

De forma análoga a lo observado en el grupo anterior, los estudiantes del Grupo 02, que manifestaron inicialmente poca inclinación hacia la PT, cuando elaboraron su propio material lo hicieron teniendo en cuenta preferentemente esta PT. Aquellos estudiantes que hallaban pertinente la $\mathrm{PH}$ en la enseñanza de los contenidos científicos, fueron más consecuentes con ello, lo cual se vio reflejado en sus propias producciones. Sin embargo, y como sucedió con el Grupo 01, los estudiantes del Grupo 02 que habían manifestado ventajas en el abordaje de las P'T y PH de forma paralela, han optado únicamente por la P'T en la elaboración de su material de enseñanza.

En la Fase final del estudio, Fase $V$, en la que se adelantaron las entrevistas para recabar información en torno a las discrepancias y coherencias obtenidas durante la investigación, se encontró que los estudiantes, profesores en formación, justificaron su desempeño en relación a los siguientes aspectos:

. Se percibe un amplio consenso en relación a que existe la necesidad de enseñar ciencias desde los niveles básicos de escolaridad. No obstante, los modelos de ciencia y de enseñanza de las ciencias que fundamentan las prácticas de estos profesores en formación son 
poco elaborados, pues existen contradicciones importantes, recurrentes y latentes, lo que pudo observarse durante todas las fases de esta investigación.

. Se considera la ciencia como una actividad humana, que conlleva altos niveles de subjetividad de los científicos, que está inmersa en contextos sociales, políticos y culturales, y que intenta dar respuesta a problemáticas específicas derivadas de su entorno. Sin embargo, las producciones propias privilegian la transmisión de contenidos científicos de forma teórica, centrada en definiciones y que se apoyan en la realización de actividades de 'refuerzo' de lo presentado como contenido temático.

. No se reconocen las diversas posibilidades que tendría el profesor, a propósito de la utilización de la Historia de la Ciencia, en particular a las diversas perspectivas que de ella se han podido caracterizar.

. Se pudo establecer una vez más la importancia que se asigna a los libros de química como referentes del saber a enseñar en la sala de clase. Así, se determinó que la fuente de información a la que se acudió para la elaboración del propio material presentaba un carácter notoriamente conceptual que soslaya la Historia de la Ciencia, lo cual fue considerado como una causa de la perspectiva teórica/conceptual (PT) de sus propias producciones.

\section{Conclusiones e implicaciones didácticas}

A partir del análisis de lo encontrado en cada una de las fases de este estudio se puede plantear que, en teoría, para los profesores en formación la Historia de las ciencias aplicada en su enseñanza y posterior ejercicio profesional docente puede tener múltiples ventajas y utilidades, relacionadas con un aumento en la calidad de los aprendizajes. Parece que en cierto modo la Historia de la ciencia puede mejorar la comprensión de los alumnos, hacer que el aprendizaje sea más significativo, que se tenga una visión más humana de la ciencia y de su progreso.

$\mathrm{Al}$ tener como referente ciertas concepciones iniciales sobre la Naturaleza y la Historia de la ciencia; diversas tendencias declaradas en torno a la pertinencia de una u otra perspectiva en los materiales propuestos; y la producción de unidades didácticas a implementar, se hace evidente la disminución progresiva de la presencia de la historia de la ciencia, esto es, el alejamiento progresivo de un modelo de ciencia problemático y dinámico hacia un modelo cada vez más próximo al modelo tradicional de ciencia dogmático. En el contexto chileno, este tipo de incoherencias entre las concepciones de los profesores y sus prácticas docentes también han sido documentadas en profesores en ejercicio, con resultados similares a los encontrados en este estudio (CONTRERAS, 2006), lo cual evidencia que estas contradicciones se mantienen a lo largo del ejercicio profesional docente.

Las causas de las incoherencias encontradas pueden deberse, según argumentan los propios estudiantes en las entrevistas realizadas, a los modelos bajo los cuales han sido formados. Parece claro que hay poca reflexión en torno al modelo de ciencia que tienen los estudiantes, y así las cosas, se aduce que por ello se tiende a reproducir lo que les han enseñado y a utilizar como referente teórico predominante los libros de texto que ellos han trabajado en los cursos precedentes. Derivado de lo anterior, puede entenderse que estudiantes y profesores tengan una visión deformada de la naturaleza de la ciencia, su objeto y método de estudio, así como de las repercusiones sociales de la ciencia, lo que puede llegar a producir una actitud de 
rechazo en la escuela, frente al estudio de las diferentes disciplinas científicas en niveles de formación superior.

A pesar del alto reconocimiento de la riqueza didáctica de la historia de la ciencia, los estudiantes tienen pocos recursos para generar nuevas propuestas, ya sea por deficiencias en su formación o por la escasez de materiales y fuentes de referencia de corte no tradicional. La limitada presencia del uso de la historia en los materiales propuestos por los estudiantes nos ha impedido incidir en una cuestión fundamental que es cómo incorporar la Historia de la Ciencia en el aula, en relación a las múltiples formas de concebirla y de los múltiples objetivos que pueden ser abordados desde cada una de ellas. No se llega a la reflexión de que, tal como señala Kragh (1989), debido a las múltiples perspectivas de la Historia de la ciencia en la actualidad, seguro que ninguno de los objetivos abarque la disciplina en su totalidad y podrá darse también que desde una sola perspectiva histórica no pudieran lograrse todos ellos. En las producciones de los profesores en formación, a las que se hace mención como Perspectiva Histórica $(\mathrm{PH})$, aparece de forma recurrente la inclusión de las biografías de los científicos, con el riesgo de convertirla en la llamada hagiografía, una Historia acrítica en blanco y negro donde se convierten algunos científicos en héroes de la ciencia. Como se estableció en el marco teórico existen diferentes aproximaciones a la historia de la ciencia, pero su uso en la enseñanza no garantiza que se transmita un modelo de ciencia naturalizada, por lo cual el profesor ha de tener una formación teórica y metodológica fundamentadas.

Las dimensiones que configuran el discurso profesional docente, las que han sido analizadas en el presente estudio: modelos de formación, concepciones metateóricas, disposición a la innovación y fuentes de referencia, han de estar estrechamente relacionadas de manera simultánea. Parece ser, y esto será interés de un nuevo estudio, que ante las dificultades propias de a lo menos una de estas dimensiones aparece reiteradamente la tendencia a replicar el modelo tradicional de ciencia.

Ante este modelo tradicional fuertemente arraigado, que constituye el punto de partida para algunos o el modelo al que recurren otros, a menudo defendido bajo argumentos que van en pro de la "eficiencia" de la enseñanza de las ciencias tomada bajo la luz reduccionista de los resultados ante las pruebas evaluativas de carácter instrumental, podemos establecer que aunque posiblemente la Historia de la ciencia no nos permita resolver problemas científicos de corte tradicional, este no debe ser el único objetivo de la educación científica, sino que además se puede promover que los estudiantes comprendan mejor la 'ciencia actual' en su contexto social, político, económico, etc, al tiempo que potenciar cambios significativos en el discurso del profesor.

\section{Agradecimientos}

Al Proyecto FONDECYT 1095149 que patrocina la comisión Nacional de Investigación Científica y Tecnológica de Chile, del que se hace parte este artículo de investigación. 
Fernández, L C.; Gatica, M. Q.; Blancafort, A. M.

\section{Referencias}

BARONA, J. L. Ciencia e historia: debates y tendencias en historiografía de la ciencia. Valencia: Seminari d'Estudis sobre la Ciència, 1994.

CAMACHO, G. J. Ley periódica: una reflexión didáctica desde la Historia de las Ciencias. 2005. 190f. Tesis (Magíster en Docencia de la Química) - Facultad de Ciencia y Tecnología, Universidad Pedagógica Nacional, Bogotá, 2005.

CONTRERAS, S. ¿Qué factores pueden influir en el trabajo de los profesores de ciencias chilenos? Revista electrónica de enseñanza de las ciencias, España, v. 5, n. 2, p. 378-392, 2006.

CUELLAR, L.; QUINTANILLA, M. La historia de la química en la reflexión sobre la práctica profesional docente: un estudio colectivo de casos. In: ENCUENTRO DE FILOSOFÍA E HISTORIA DE LA CIENCIA DEL CONO SUR, 6., 2008, Montevideo. Actas... Montevideo: AFHIC, 2008. Disponible en: http:/ /afhic.easyplanners.info/ programa/buscar.php?id_tl=354. Acceso en: 21 ago. 2010.

FURIÓ, C. Las concepciones alternativas del alumnado: dos décadas de investigación resultados y tendencias. Revista Alambique - Didáctica de las ciencias experimentales, Barcelona, n. 7, p. 7-17, 1996.

GARCÍA, A. Aportes del estudio histórico de instrumentos científicos a la formación del profesorado de ciencias. 2003. 176f. Tesis (Maestría en Didáctica de las Ciencias Experimentales y las Matemáticas) - Universitat Autonoma de Barcelona, Barcelona, 2003.

GIERE, R. La explicación de la ciencia: un acercamiento cognoscitivo. México: Concejo Nacional de Ciencia y Tecnología, 1992.

GIL, D.; CARRASCOSA, J.; MARTÍNEZ TERRADES, F. El surgimiento de la didáctica de las ciencias como un campo específico de conocimientos. Educación y Pedagogía, Antioquia, v. 11, n. 25, p. 15-65, 1999.

IZQUIERDO, M. Fundamentos epistemológicos. In: PERALES, F. J.; CAÑAL, P. (Eds.). Didáctica de las ciencias experimentales: teoría y práctica de la enseñanza de las ciencias. Madrid: Alcoy, 2000. p. 35-64.

.; ALIBERAS, J. Pensar, actuar i parlar a la clase de ciències. Barcelona: Universitat Autónoma de Barcelona, 2004.

. VALLVERDÚ, J.; QUINTANILLA, M.; MERINO, C. Relaciones entre la historia y la filosofía de la ciencia II. Alambique - Didáctica de las ciencias experimentales, Barcelona, v. 48, n. 1, p. 78-91, 2006.

KRAGH, H. Introducción a la historia de la ciencia. Barcelona: Crítica, 1989.

LABARRERE, A.; QUINTANILLA, M. La solución de problemas científicos en el aula. Reflexiones desde los planos de análisis y desarrollo. Pensamiento Educativo, Santiago de Chile, v. 30, p.121-137, 2002. 
La importancia de la historia ...

MATTHEWS, M. R. Historia, filosofía y enseñanza de las ciencias: la aproximación actual. Enseñanza de las Ciencias, Barcelona, v. 12, n. 2, p. 25-277, 1994.

PORLÁN, R. Pasado, presente y futuro de la didáctica de las ciencias. Enseñanza de las Ciencias, Barcelona, v. 16, n. 1, p. 175-185, 1989.

PORLÁN, R.; MARTÍN DEL POZO, R. Cómo progresa el profesorado al investigar problemas prácticos relacionados con la enseñanza de las ciencias? Alambique - Didáctica de las ciencias experimentales, Barcelona, v. 48, n. 1, p. 92-99, 2006.

QUINTANILLA, M. Identificación, caracterización y evaluación de competencias científicas desde una imagen naturalizada de la ciencia. In: QUINTANILLA, M.; ADÚRIZ-BRAVO, A. (Eds.). Enseñar ciencias en el nuevo milenio. Santiago de Chile: Ediciones Pontificia Universidad Católica, 2006. p. 17-42.

.; IZQUIERDO, M.; ADÚRIZ-BRAVO, A. Characteristics and methodological discussion about a theoretical model that introduces the history of science at an early stage of the experimental science teachers' professional formation. In: INTERNATIONAL HISTORY, PHILOSOPHY, SOCIOLOGY \& SCIENCE TEACHING CONFERENCE, 8., 2005, Leeds. Proceedings... Leeds: University of Leeds, 2005. Disponível em: <http:// www.ihpst2005.leeds.ac.uk/papers/Quintinilla_Izquierdo_Aduriz-Bravo.pdf >. Acesso em: 10 dez. 2006.

.; LABARRERE, A.; DÍAZ, M.; CAMACHO, J.; CUELLAR, L.; RAVANAL, E. El inventario de ideas previas (KPSI) como un instrumento de regulación de los procesos de desarrollo profesional de docentes de ciencias naturales en ejercicio. Pensamiento Educativo, Santiago de Chile, v. 22, n. 1, p. 97-114, 2007.

SANMARTÍ, N. Necesidad de formación del profesorado en función de las finalidades de la enseñanza de las ciencias. Pensamiento Educativo, Santiago de Chile, v. 30, n. 1, p. $35-60,2002$.

SOLSONA, N.; QUINTANILLA, M. Reflexions i propostes per al debat educatiu - diàctic entorn a la història de la ciència. In: JORNADA SOBRE LA HISTÒRIA DE CIÈNCIA I L'ENSENYANMENT, 2., Barcelona. Actas... Barcelona: Societat Catalan d'Història de la Ciència, 2005. 1 cd-rom.

SOLSONA, N. Mujeres científicas de todos los tiempos. Madrid: Talasa, 1997.

TOULMIN, S. La comprensión humana: el uso colectivo y la evolución de los conceptos. Madrid: Alianza, 1977. 